\title{
An Efficient Healthcare Monitoring and Pre-Emptive Ambulance Control Through Traffic Signals
}

\author{
Vasanthan $V^{1}$, Teja Sai Vignesh $D^{2}$, Selvabharathi $S^{3}, S$ Sree Subha ${ }^{4}$ \\ 1,2,3\&4 Department of Information Technology, Rajalakshmi Engineering College \\ Chennai, Tamilnadu, India \\ Email: vasanthan.v.2016.it@ rajalakshmi.edu.in $1^{1}$ tejasaivignesh.d.2016.it@ rajalakshmi.edu.in ${ }^{2}$ \\ selvabharathi.s.2016.it@rajalakshmi.edu.in3 sreesubha.s@rajalakshmi.edu.in ${ }^{4}$
}

\begin{abstract}
Monitoring the pulse, heartbeat rate and oxygen levels of a person in an ambulance and then shifting the person to the emergency ward and then continuing the monitoring is what is usually done. The monitoring is stopped when the person is moved from the ambulance and admitted in the emergency ward. This is a big disadvantage because there is a chance of a drop in oxygen or pulse levels at any particular time. We eliminate this disadvantage by installing a pulse sensor, temperature sensor, GPS , pressure sensor, and gas sensor in the stretcher. All these can be connected to a person's body and can be monitored continuously. All these data are displayed on a webpage using IoT. The same stretcher can be used in the emergency ward and the data of the person is not lost at any time period. Using nrf sensors, the ambulance makes communication with the traffic signals located on the ambulance's way and makes it turn green until the ambulance passes through it. Therefore the ambulance can reach the hospital soon.
\end{abstract}

Keywords- IoT, GPS, pressure, pulse, mobile app, temperature, NRF

\section{INTRODUCTION}

The ambulance plays a significant role in saving lives. There are a lot of hindrances in the way of an ambulance reaching the hospital. One such considerable hindrance is traffic. The traffic in the way of an ambulance needs to be cleared. To resolve this problem, many technologies have been introduced. One such technology is the use of RFID. But the problem with the RFID's is that communication between ambulance and traffic signal occurs only when the ambulance is too close to the traffic signal. To encounter this drawback, we are using Nrf sensors (Nearfar communication) in our proposed system. This nrf sensor is attached to the ambulance and with the traffic signals. When the ambulance comes near the traffic signal, the corresponding traffic signal turns green until the ambulance passes through it. Even in a 4-way junction road, the corresponding traffic signal is turned green and other traffic signals are not disturbed. Thus the proposed system helps the ambulance to reach the hospital on time.

Another problem that happens in an ambulance is monitoring the vital data such as heartbeat rate, temperature, the pressure within the ambulance and the problem in this is the data is not continuously monitored. The data is not monitored when the person is shifted from the ambulance to the emergency ward. To encounter this problem, we have come up with a solution wherein all the sensors like the heartbeat rate sensor, pressure sensor,

Vol. 4 (5), November 2020, www.ijirase.com the humidity sensor is all situated in the stretcher itself. And this stretcher is carried out to the emergency ward. Such that there is continuous monitoring of data, we ensure that the data is not lost in the middle.

Internet of Things (IoT) is a rising technology. Using IoT, we enable a real-time data transfer of the data monitored in the ambulance. This helps the doctors in the hospital to do the suitable treatment according to the levels of data. This also allows the doctors to guide the paramedics most efficiently.

\section{II.RELATED WORK}

Aprita K.M et al. proposed a system [1] that aims to collect and send the patient's information to the hospital prior to the patient. Apart from the basic devices present inside most ambulances like emergency medical kit, two types of stretchers, glucometer, portable oxygen cylinder, defibrillator, ECG, and ventilator also use various sensors. This system continuously monitors the patient's vital health parameters. This system reduces the time wasted in fulfilling hospital formalities.

Syed Misbahuddin et al. proposed a system [2] that stores real-time data locally for a short time for the observation of on-board paramedics. The data is also displayed graphically on an LCD screen of the host computer. The hospital staff can scrutinize the patient's health status generated from the ambulance in real-time web page while the patients are on the way to the hospitals. The doctors can view the patient's health 
conditions and communicate with the ambulance's paramedics to ossify them - the proposed system using the Internet of Things (IoT) for real-time data transfer. The proposed system does not have a preemptive traffic control system.

Qurrat UI Ayan et al. proposed a system [3] that monitors for emergency patients even during transportation to a hospital in an ambulance. A low-power wireless wearable monitoring device is implemented using an embedded system, sensors, IoT and GSM technology. This project performs three main functions. At first, it monitors the patient's health then tracking the ambulance in which the patient is present and the third one is to send the above two information to the Hospital or Doctor using GSM and the values of the vital parameters to the cloud using Wi-Fi Technology.

Elangeswari et al. proposed research [4] in which first, a semantic data model is submitted to store and interpret IoT data. A resource-based data pervade model is then designed to acquire and process IoT data far and wide to improve the accessibility to IoT data resources. Finally, an IOT based emergency monitoring system and using it for quick medicare of patients is developed.

S. Dhivya et al. proposed a system [5] to control traffic light signals using IoT. It helps the ambulance to reach the hospital soon and also saves the patient life. It is also used to send the ambulance's location to get the traffic signal for prior arrangements to manage the traffic. The project controls the ambulance's traffic lights and makes the freeway automatically without any disturbance to the public. The project is used to save time delay in the most efficient way to save a life. This system uses a timer and Arduino for traffic signal control. Problem with traffic signal control may occur in a 4-way junction.

Irin Sherly et al. proposed a system [6] that connects the ambulance with the IoT with biomedical sensors like the heartbeat rate sensors, temperature sensors, and ECG sensors that will sense and detect the injured person's health conditions and update this information to the nearby hospital server. And also, this system has a finger print scanner that takes the fingerprint of the patient and sends to the hospital server and the server will search for the patient's fingerprint in the cloud and fetch the personal details of the person, then automatically registers the patient's admission form
S.N.Sivaraj et al. proposed a system [7] that consists of two important things. At first, the patient's basic information and status are collected in the ambulance by the means IoT (Internet Of Things) and make available to the hospital before the ambulance reaches the hospital. The second section is to control traffic lights from the ambulance and makes clearance for its path automatically. Thus this project allows us to save the time of major delay and improved efficient and economical manner.

Deepali Ahir et al. proposed a system [8] with Intelligent Traffic Control System for an ambulance. The proposed system clears the traffic congestion, turning all the signal in red lights to green on the ambulance's path. The system consists of an android application that log the details of the ambulance on its network. Suppose the ambulance struck on its way in case of an emergency situation. In that case, the system sends an emergency message to the traffic signal server and the direction where it wants to travel. With this, the current position with the Global Positioning System's help. Based upon the current position of the ambulance, the nearest signal is identified. The particular signal is made green till the ambulance crosses it, latter it becomes normal mode. In this way, it becomes a lifesaver project as it saves time during an emergency by controlling the traffic lights. The problem occurs when the ambulance crosses a 4-way junction.

Syed Mohd Faisal Malik et al. proposed a system [9] that helps navigate the traffic by using a robust algorithm and round-robin algorithm to find the optimum path. Raspberry $\mathrm{Pi}$ is used to manage all components collectively and individually. Total traffic in a zone is determined by the help of IR sensors, which used RFID to distinguish high preference vehicles such as ambulance and VIPS from day to day traffic. 
III. PROPOSED METHODOLOGY

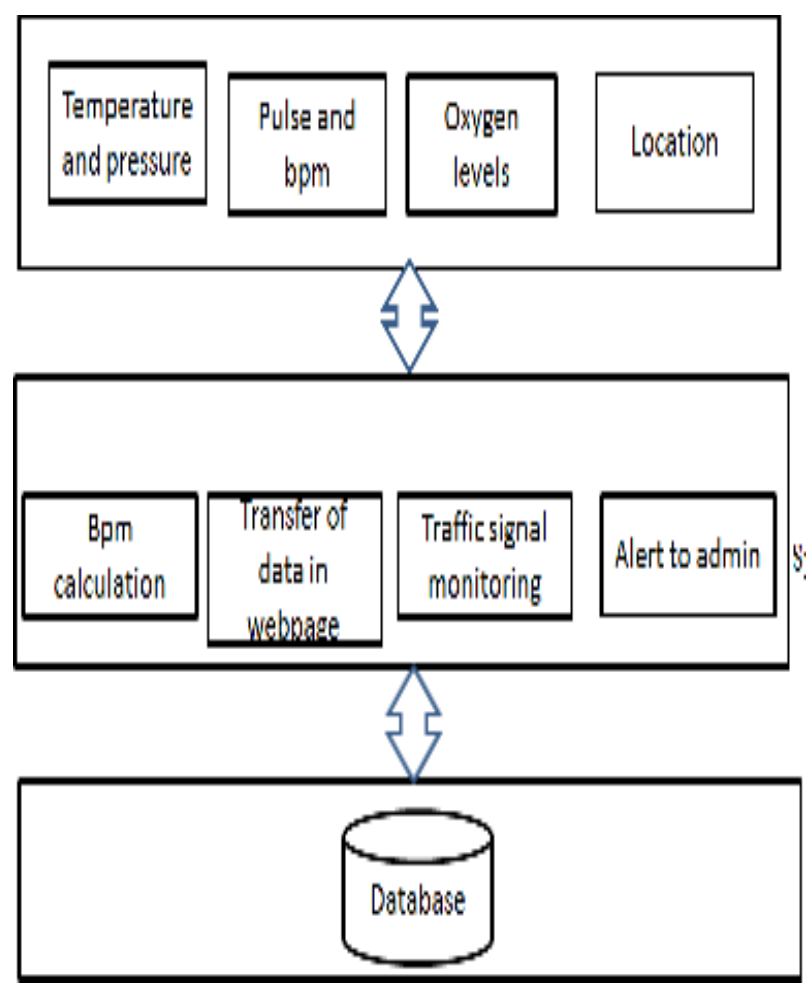

Figure 1: Architecture diagram

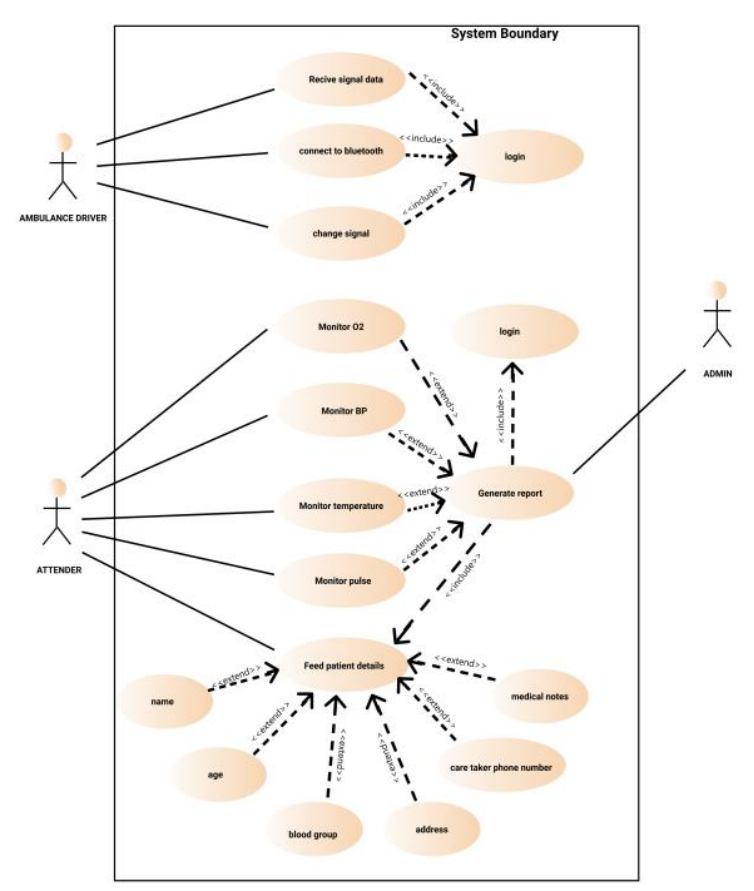

Figure 2: Use Case diagram

\section{a. Monitoring of vital data}

The vital data such as temperature, heartbeat rate and pressure of a person in an emergency is measured using the heartbeat sensor, pressure sensor, temperature sensor, respiratory sensor. These sensors are attached to the stretcher itself in the ambulance. The same stretcher is used in the hospital too.

\section{b. Real-time transfer of data using IoT}

Using IoT, we display the vital data in a webpage dynamically. The values get updated automatically without refresh. This technique enables the doctor to know about the patient before the patient arrives in the hospital. This also allows efficient communication between the doctor and the paramedics.

\section{c. Pre-emptive ambulance control}

A mobile app with IoT controllers which continuously sends/receive the next nearest signal and enable the driver to control the movement by changing the traffic lights; at this moment, the precedence of traffic lights are given to the ambulance driver; once he passes away the signal, he may turn it to normal. Therefore the ambulance can reach the hospital soon.

Table 1: Precedence Table

\begin{tabular}{|l|l|}
\hline Ambulance & 1 \\
\hline Normal & 0 \\
\hline
\end{tabular}

We have a kit for the ambulance driver and install a kit in the traffic signal zone. The kit, which the ambulance driver has, contains NRF and Bluetooth module with a UART micro-controller(Arduino-Uno) controlling them; it allows the ambulance driver to connect his mobile device to the kit.

The driver will have an app, which can show up the signal, which is upcoming to him. And allow him to select the way he wants to go.

On selecting the way, the traffic light will be changed; as we have another NRF and Arduino kit in the signal zone, this kit will becon the available number of signal and notify the kit in the ambulance to the driver' mobile app. 
In order to select the right signal, we feed every signal name with the help of a compass, the signal facing its direction is named correspondingly, like north, south, east, west. With this, the ambulance driver can find the right signal; he wants to turn green, as he too has a compass showing the direction.

We are also concerned about the safety of this wireless Transmission/Reception, which we don't want to be interfused with other devices, So we apply the Caesar cipher Encryption technique. The traffic signal located on the ambulance's way is turned on green till the ambulance passes through .

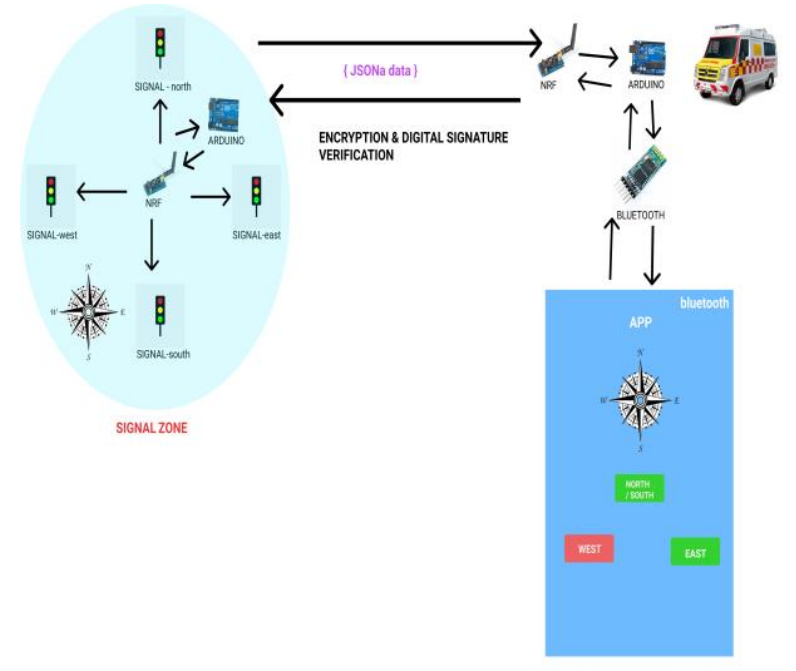

Figure 3: Mind Chart

\section{d. Encryption Algorithm for Transmission/Reception}

It is a type of substitution cipher in which each letter in the plaintext is shifted a certain number of places down the alphabet.

For encrypting:

$$
C(x)=(x+k) \bmod 26
$$

For Decrypting:

$$
\mathrm{P}(\mathrm{x})=(\mathrm{x}-\mathrm{k}) \bmod 26
$$

Where $C(x)$ is the ciphertext

$$
\mathrm{P}(\mathrm{x}) \text { is the plaintext }
$$

$\mathrm{x}$ is the plaintext

$\mathrm{k}$ is the number of shift

\section{RESULTS}

\section{a. Monitoring of vital data}

The temperature, heartbeat and pressure sensor are attached to the stretcher and the patient. The data coming out of these sensors is collected and sent to the web server, which is further given to the webpage. This web page is accessible to the hospital, with which the doctor can know the patient's live health condition and assist the paramedics. This technique is known as the Internet Of Things(IoT).

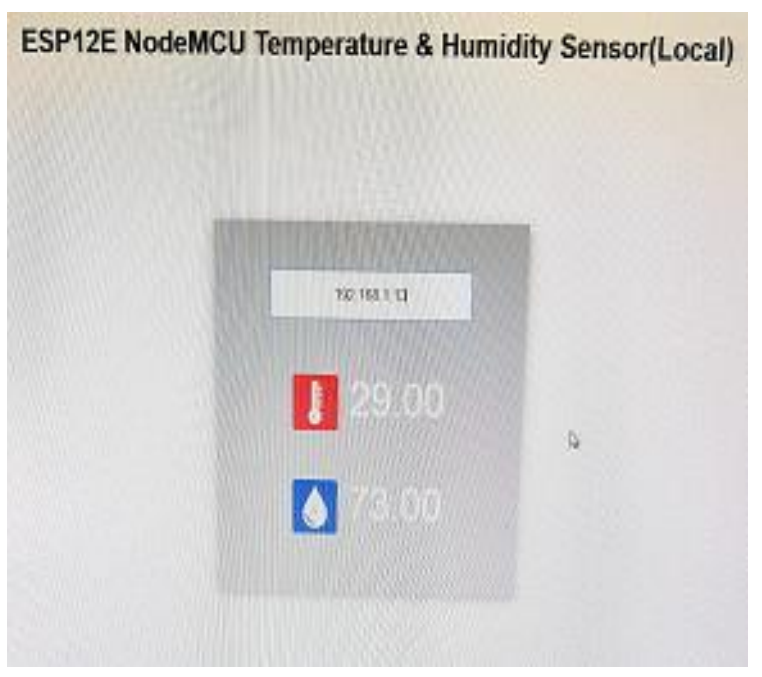

Figure 4: Monitoring of data in the webpage

\section{b. Pre-emptive ambulance control}

We have installed two kits, one will be in an ambulance and another will be installed in every signal. The kit in the ambulance contains a Bluetooth module, NRF, RFID sensor. Bluetooth module helps connect the kit to the app we have build, NRF helps in communicating with the signal for changing the lights. RFID is used to ensure the right user is accessing the kit. At first, the driver used to connect with the kit's Bluetooth named "ambulance" once the driver is paired to the device, he can switch to the app.

Here he will be checked for permission and Bluetooth connectivity. Now the driver will be asked to keep his RFID tag to verify himself, if the right user, then allowed into the app, if the unauthorized user then an alert sound and an emergency message is sent to the control room or any other emergency department. After that, he will be allowed to select the kit's Bluetooth and connect to it; if 
he chooses the wrong Bluetooth device, a prompt with a warning message will be displayed. The user is then allowed to the main page where he can see the Bluetooth device and its MAC Id he is connected to, a compass to select the right signal, and four buttons, which is used to turn the signal green.

The kit in the signal contains an NRF sensor, which keeps on bacon the available number of signals. The signals were named according to the compass direction in that zone so that the driver can find the right signal.

On receiving the signal data to the app, only the available signal button is enabled by which the driver can be notified; then the driver can turn the signal green by clicking the button of his way; once he crosses the signal, he can click on the same button to turn the signal to the normal mode.

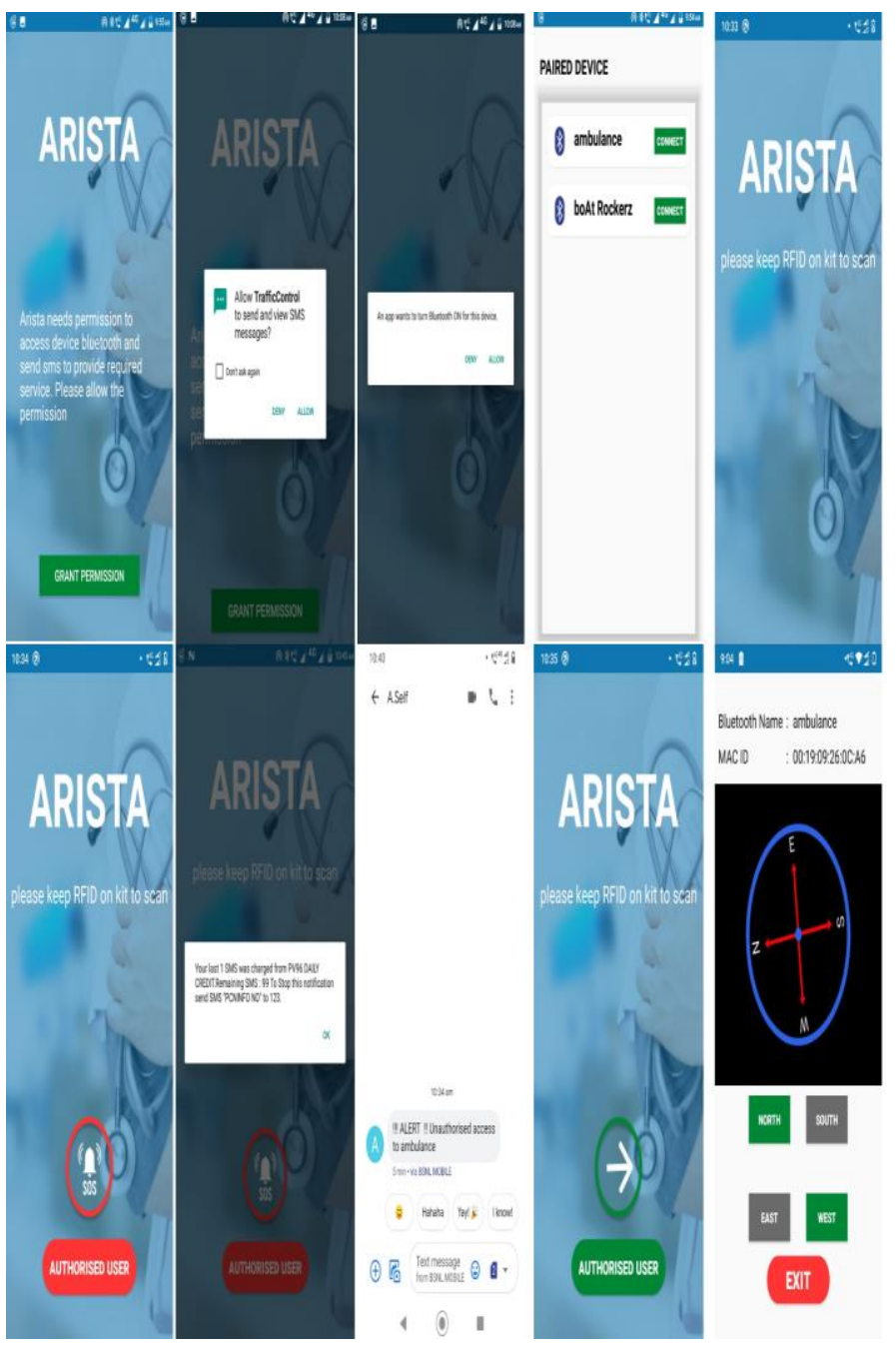

Figure 5: Android app UI

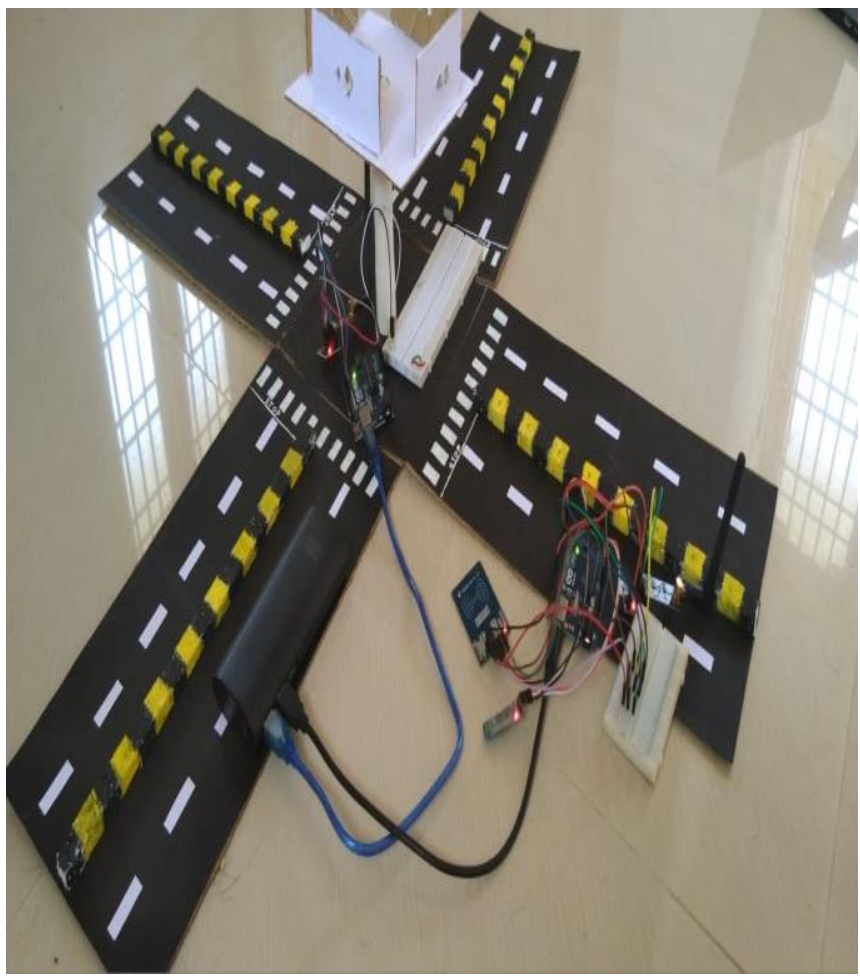

Figure 6: Working Model

\section{CONCLUSION}

The proposed system helps monitor the vital data of a patient in the ambulance and enables real-time data transfer of critical data to the hospital. The essential data is also displayed on the webpage and the values are updated dynamically. This allows the doctor to know about the patient before the patient arrives in the hospital, suitable treatment can be given to the patient. Our proposed system also enables efficient communication between the doctor and the paramedics. Our proposed system also enables the patient's fast transportation by using NRF sensors(near far communication) that helps control traffic signals. The traffic signal in the way of the ambulance turns green.

\section{REFERENCES}

[1] Aprita K.M, K.S Asha , T. Ashwan , Janvi Maddi, M.P Sunil, "Health monitoring system for ambulance ", 2019.

[2] Misbahuddin, S., Zubairi, J. A., Alahdal, A. R., \& Malik, M. A. (2018). IoT-Based Ambulatory Vital Signs Data Transfer System. Journal of Computer Networks and Communications, 2018.

[3] KASENEGALA, L. (2016). AMBULANCE TRACKING WITH PATIENT HEALTH MONITORING SYSTEM USING GPS AND GSM SYSTEMS (Doctoral dissertation).

[4] Elangeswari , R. Poorni, R. Rashmi , M. Varalakshmi ," Emergency medical services in Internet of Things ", International Journal of engineering Technology in 
Computer Science and electronics, volume Issue 3 , April 2016.

[5] S. Dhivya , M. Palani , S. Saravanakumar, S.Venkatesh , M. Vikhneswaran, "IoT based traffic signal control for ambulance ", International Journal of Pure and Applied Mathematics, volume $118,2018$.

[6] S. Irin Sherly, A. Maria Soitham Princy, S. Monisha , K. Nandhini ," Smart ambulance rescue system with patient health monitoring using IoT, International Journal of Advanced Research in Science, Engineering and Technology , Vol 6, Issue 2, February 2019.

[7] Sivaraj, S. N., Vigneshwaran, K., Vigneshwaran, S., \& Priyan, M. (2017). Iot Ambulance With Automatic Traffic Light Control. International Journal of Industrial Engineering (ICRTECITA2017) Special issue.

[8] Ahir, D., Bharade, S., Botre, P., Nagane, S., \& Shah, M. (2018). Intelligent Traffic Control System for Smart Ambulance. Int. Res. J. Eng. Technol, 5(6), 5.

[9] Nafis, M. T., \& Khan, M. H. (2018). IoT ENABLED TRAFFIC CONTROL MODEL USING RASPBERRY PI. International Journal of Advanced Research in Computer Science, 9(3), 157. 